\title{
OXYUROSE DES VOIES GÉNITALES CHEZ LA FEMME. DISCUSSION DU CYCLE ENDOGÈNE DE L'OXYURE
}

\author{
(A propos de trois cas de lésions vermineuses \\ de l'ovaire et de ses annexes)
}

Par J.-M. DOBY, M.-L. CHEVREL, B. RAULT et L. DAULEUX

Nous avons eu l'occasion récemment d'observer trois cas de lésions provoquées par des Oxyures au niveau de l'ovaire et de la trompe. Il convient de préciser en premier lieu que l'oxyurose de ces trois patientes n'avait pas été cliniquement soupçonnée et que les découvertes opératoires avaient conduit à la suspicion de la tuberculose. Les lésions sont de deux types : soit diffuses, soit nodulaires. Les premières se présentent en nappes inflammatoires lympho-plasmocytaires, avec un nombre variable de leucocytes polynucléaires et quelques plages de nécrose. Celles-ci contiennent des éléments parasitaires : œufs, coques vides, jeunes larves. Les secondes formations consistent en des nodules de type épithélioïde. Leur masse est, en effet, constituée par des histiocytes à type épithélioïde avec des cellules géantes. Leur centre est souvent nécrosé et, dans beaucoup d'entre eux, la nécrose non homogène renferme un ou plusieurs éléments parasitaires. D'autres aspects peuvent être relevés. Il existe toutes les formes de passage entre le nodule compact sans nécrose et le nodule massivement nécrotique (1).

Dans les zones de nécrose, les éléments parasitaires sont souvent associés à de nombreux cristaux de Charcot-Leyden. Ces éléments consistent en œufs et en jeunes larves d'Enterobius vermicularis. Les œufs, de 45 à $55 \mu$, à coque lisse, épaisse et réfringente, avec leur asymétrie caractéristique en coupe longitudinale, présentent tous les stades de développement : quelquefois simplement segmentés, le plus souvent embryonnés, les uns au stade gyriniforme, les autres au stade larviforme. Plusieurs ont été fixés en cours d'éclosion, l'embryon larviforme déjà partiellement engagé dans la per-

(1) Une étude détaillée histopathologique est donnée par ailleurs (Chevrel et coll., 1961). 
foration au niveau de l'aire poreuse. De nombreuses coques vides peuvent être observées, ainsi que des jeunes embryons libres, dans les tissus, et, parfois même, des larves nettement plus développées dont la cuticule montre déjà une striation transverse marquée. Les coques vides se présentent à divers stades de destruction. Elles sont en effet envahies par des cellules du tissu environnant, éclatent et se fragmentent avant d'être totalement lysées. Il n'a pas été observé de vers adultes.

Dans l'un des tro:s cas, oà il y avait de nombreux granulomes, il semble que les dépôts d'œufs, au niveau de chacun d'eux, par les

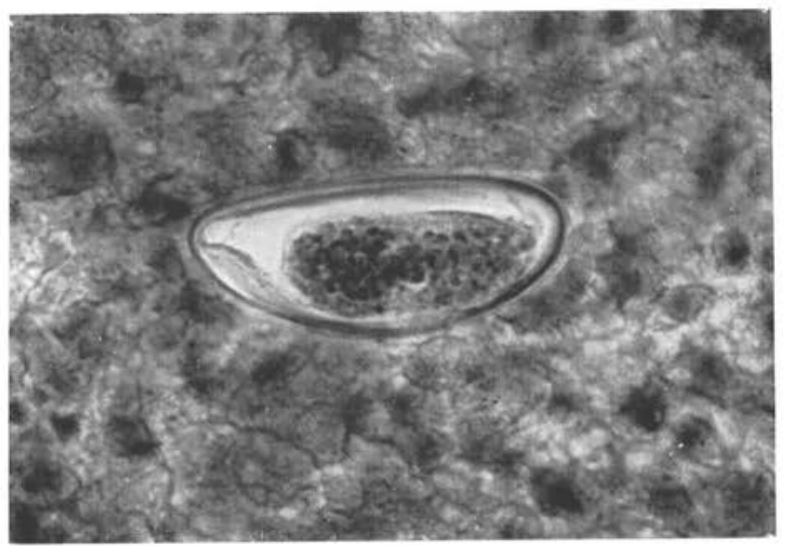

Рнот. 1. - Euf d'oxyure embryonné au stade gyriniforme

femelles en migration, n'aient pas été effectués simultanément. En effet, suivant les granulomes, nous avons pu observer, en éléments parasitaires dominants :

— soit des œufs embryonnés à divers stades de développement ;

- soit des coques vides intactes et des larves;

- soit des coques vides en voie de destruction.

Enfin, dans certains nodules, tout résidu parasitaire a disparu dans la nécrose (2).

(2) On sait qu'il suffit de quelques heures pour la maturation des œufs (passage du stade gyriniforme au stade larviforme) et pour leur éclosion après ingestion. Mais ceci n'est valable que pour l'œuf se trouvant dans les conditions normales de son cycle évolutif. Lorsque, au contraire, il se trouve dans des conditions inhabituelles, comme c'est le cas ici, il est impossible de fixer des délais. Expérimentalement, cette évolution peut être lente et demander jusqu’à 20 jours. Par ailleurs, il est également difficile d'apprécier le temps nécessaire à la lyse des coques vides. Par conséquent, on ne peut estimer ici les intervalles de temps ayant séparé les dépôts d'œufs aux différents points de ponte. 
Quelle est l'origine de ces éléments parasitaires égarés ?

Deux hypothèses ont été envisagées pour expliquer la présence dans de tels granulomes d'œufs d'Oxyure ou de femelles en migration dans la cavité abdominale : soit par remontée des femelles, issues du tube digestif, le long des voies génitales ; soit par traversée directe au travers de la muqueuse intestinale.

En ce qui concerne la seconde, il n'est pas rare en effet de trouver des Oxyures enfoncés profondément dans la muqueuse (Brumpt et Lecène, 1909 ; Railliet, 1911 ; Cauchemez, 1929). Ceci s'observe fréquemment au niveau de l'appendice. Deblock (1952) a ainsi noté

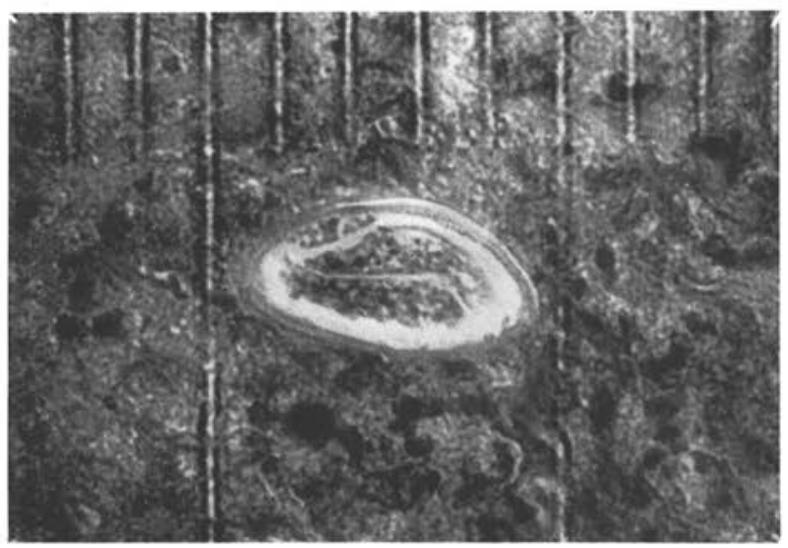

Рнот. 2. - Euf d'oxyure embryonné au stade larviforme (l'échelle micrométrique en superposition est valable pour toutes les photos; une divi$\operatorname{sion}=10 \mu$.

les diverses modalités de cette localisation : «Lorsque la muqueuse est macroscopiquement saine, il est possible de rencontrer des Oxyures (uniquement femelles dans les observations de cet auteur), dont l'extrémité céphalique est enfoncée plus ou moins profondément dans son épaisseur. Parfois, les Oxyures colonisent une muqueuse fortement délabrée, se logeant dans une sorte de cheminement sous-muqueux et communiquant avec la lumière appendiculaire par un large orifice ; dans certains cas, même, on peut les trouver en contact direct avec la tunique musculeuse. 》Deblock, cependant, bien qu'ayant examiné macroscopiquement et histologiquement 132 appendices, note n'avoir jamais rencontré d'Oxyures ayant pénétré dans la musculeuse. Cette pénétration, suivie parfois d'enkystement du ver à ce niveau, a pourtant été signalée par cer- 
tains (Vuillemin, 1902 ; Liengmé, 1932). Ce phénomène a pu, par ailleurs, être observé chez d'autres espèces animales que l'homme, parasitées par des Cxyuridés divers : chez le lièvre, parasité par Oxyuris ambigua (Penso, 1932); chez le chimpanzé, parasité par Enterobius anthropopiteci (Weinberg, 1904; Troisier et Deschiens, 1950). La perforation totale de la paroi digestive semble même, dans des circonstances assez exceptionnelles, pouvoir se réaliser. Dès 1902, Vuillemin avait envisagé cette possibilité : «Il est démontré que les femelles attaquent la muqueuse intestinale et peuvent traverser une épaisseur de tissu d'au moins deux centimètres. Ce trajet suffit pour les amener, soit dans la cavité péritonéale, soit dans le tissu conjonctif sous-cutané... » (Cet auteur envisageait également la possibilité d'une arrivée dans la cavité abdominale par remontée, chez la femme, des voies génitales).

Ainsi, Biljmer (1946) rapporte un cas humain où de très nombreux Oxyures parasitaient toute l'épaisseur de la paroi intestinale et dont un certain nombre avait franchi les tuniques musculeuses, à la faveur d'ulcérations, préexistantes il est vrai, de la muqueuse.

Nul doute, par conséquent, que l'Oxyure puisse traverser la paroi entière du tube digestif et pénétrer ainsi dans la cavité péritonéale, où il continuerait ses migrations pendant un certain temps et finirait par mourir, comme dans le cas décrit par Whitelocke (1914).

Toutefois, un fait nous amène à penser que cette voie doit être tout à fait exceptionnelle et que la localisation péritonéale d'Oxyures a pour origine, dans la très grande majorité des cas, une remontée par les voies génitales. En effet, dans la littérature, tous les cas de granulomes abdominaux par Oxyures, à l'exception, à notre connaissance du moins, de celui récent de Symmers (1957) (localisation au niveau de la prostate), ont été observés chez la femme. La fréquence comparativement plus élevée des explorations abdominales chez la femme que chez l'homme ne suffit pas à expliquer cette remarque.

Cette remontée des Oxyures dans les voies génitales femelles n'a d'ailleurs rien d'extraordinaire. En effet, on sait que l'Oxyure, contrairement à ce qui s'observe pour les autres Nématodes intestinaux de l'homme, ne pond pas ses œufs dans la lumière intestinale, au sein même de la masse fécale, mais les dépose à la marge de l'anus, dans les replis de la muqueuse, en dehors des périodes de défécation. Les mécanismes de cette incitation à une migration descendante des femelles gravides nous échappent encore. C'est d'ailleurs la présence et les mouvements de ces femelles gravides à la marge de l'anus qui sont à l'origine du prurit anal, vespéral et 
nocturne, si fréquemment observé dans cette parasitose. Si les femelles, pour la ponte, restent habituellement au niveau de l'orifice anal même, il n'est pas rare qu'un certain nombre d'entre elles, à la faveur de l'humidité et de la chaleur du sillon périnéal, migre dans un rayon de plusieurs centimètres (Bozicewich et Brady, 1938). Chez les fillettes et les femmes, elles peuvent ainsi gagner la vulve. Ainsi, Koch (1925) réussit à observer l'arrivée, au niveau de l'orifice génital, de 16 femelles d'Oxyures sur 43 sorties de l'anus deux heures plus tôt et ayant cheminé le long du sillon périnéal. La ponte de ces femelles peut alors s'effectuer à ce niveau et il est fréquent d'y récolter, en utilisant une méthode appropriée, telle, par exem-

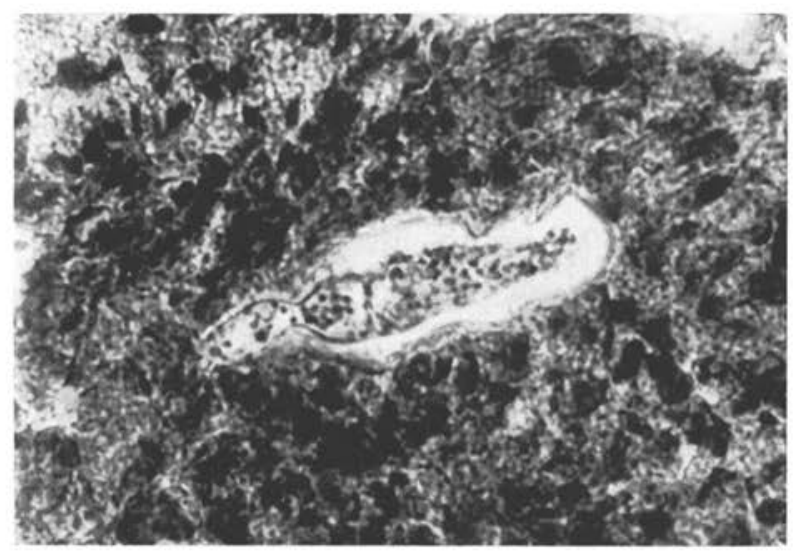

Рнот. 3 - Embryon en train de quitter l'enveloppe de l'œuf

ple, la technique de Graham à la cellophane adhésive, des œufs embryonnés d'Oxyures. Chez les fillettes parasitées, la recherche des œufs d'Oxyure est fréquemment positive au niveau de la vulve : $31 \%$ des porteuses pour Brady et Wright (1939), 80 \% pour Jacobs (1942), $88 \%$ pour Quintanar (1943), $85 \%$ pour Coutelen et coll. (1950).

Beaucoup d'auteurs ont pensé que les femelles mouraient et se desséchaient rapidement après la ponte au niveau de l'anus, II semble toutefois qu'un certain nombre d'entre elles soit susceptible de n'éliminer qu'une partie de leurs œufs et de remonter ensuite dans le tube digestif (Madsen, 1945). Une remontée analogue, mais dans les voies génitales, peut s'opérer alors que les femelles se trouvent au niveau de la vulve. Cette pénétration active fut d'ailleurs notée 
par Koch, au cours de l'observation précitée. Un entrainement passif dans la cavité vaginale peut également être envisagé, chez la femme, à la suite de rapports sexuels. Quoi qu'il en soit, des femelles d'Oxyure peuvent être retrouvées dans le vagin. Dès 1880, Vau. ghan décrivait ainsi un cas de vulvovaginite grave dû à des Oxyures chez une fillette. De même, Spitzer (1892) obtenait un peloton d'Oxyures à la suite d'un lavage vaginal chez une fillette présentant des pertes purulentes. Dans un cas analogue, Heller (1903) observait des œufs d'Cxyures dans la cavité vaginale.

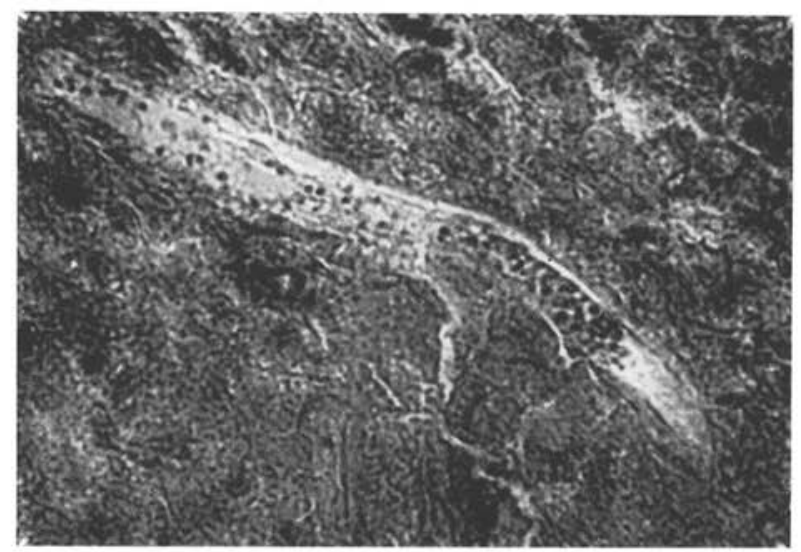

Рнот. 4. - Jeune larve dans une zone de nécrose

De là, les femelles peuvent gagner la cavité utérine. En 1860, Vix signalait déjà la présence d'œufs embryonnés d'Oxyure dans le mucus utérin. De mème, Bénédetti (in Leuckart, 1876) relevait la présence d'Oxyures adultes entre le placenta et la paroi utérine. En 1899, Simons observait in situ deux Oxyures dans le canal cervical d'une femme présentant de petites hémorragies.

Enfin, la migration peut se poursuivre, puisque Tschamer (1919) a trouvé des Oxyures vivants dans une trompe utérine venant d'être prélevée chirurgicalement. Chiari (1928) faisait plus tard la même observation. La chute dans la cavité abdominale est alors facile et des Oxyures enkystés à proximité immédiate du pavillon ont été ainsi mis en évidence par Marro (1901), Schneider (1904), Wu (1935), etc..., parfois même plus loin [Froriep (1938) : dans le repli vésico-utérin ; Kolb (1902) et Strada (1907) : dans le cul-de-sac de Douglas $]$.

ANN. de Parasitologie, T. XXXVI, $\mathrm{N}^{\circ} 1-2 .-1961$. 
La présence, au sein de ces nodules, d'œufs embryonnés à divers stades de développement, d'œuf́s en train d'éclore, d'embryons libres et même de jeunes larves ayant commencé leur évolution, nous amène à évoquer de nouveau ici la possibilité d'un cycle endogène de l'Oxyure.

Depuis de nombreuses années déjà, le cycle évolutif de l'Oxyure a été élucidé : les femelles gravides gagnent la portion terminale du tube digestif, sortent par l'anus et déposent dans les replis de la muqueuse anale des œufs embryonnés parvenus au stade gyriniforme. En quelques heures, ces œufs atteignent le stade larviforme et sont alors infectants. L'infestation de l'homme se fait par ingestion, soit directement, soit après inhalation avec des poussières. Ces œufs éclosent et donnent des larves qui se développent dans l'intestin grêle, sans effectuer de migrations hors du tube digestif. C'est là le cycle classiquement reconnu. Cependant, d'autres cycles ont été envisagés pour tenter d'expliquer la ténacité et l'intensité de certaines infections vermiculaires. Ainsi, pour certains, les œufs déposés à la marge de l'anus pourraient y éclore et les jeunes larves seraient alors capables de franchir le sphincter anal et de remonter ensuite jusque dans une partie plus supérieure du tube digestif : c'est la théorie de la « rétroinfection » de Schüffner et Swellengrebel (1949). A l'appui de cette théorie, la présence de coques vidées de leur contenu à la marge de l'anus a été observée (Schüffner, 1949). De plus, expérimentalement, ces auteurs ont pu réaliser l'infestation de volontaires, préalablement reconnus indemnes d'oxyurose, par application sur la région périanale de larves issues d'œufs soumis à une digestion artificielle.

D'ailleurs, dès 1925, Lorentz avait déjà émis l'hypothèse que les œufs étaient capables d'évoluer sur la marge de l'anus parmi les parcelles de matières fécales. Il attribuait en partie le prurit à l'éclosion de ces larves.

D'autres auteurs sont allés jusqu'à envisager une multiplication de l'Oxyure se réalisant entièrement à l'intérieur du tube digestif : c'est la théorie du cycle endogène de Penso (1932 et 1933). Cet auteur pense que «les Oxyures sont capables d'accomplir chez le même hôte, quoique en des sièges différents, toute leur évolution : la phase adulte et la période d'accouplement dans la lumière intestinale, la période de ponte pour la femelle et la phase embryonnaire de l'œuf dans la paroi de l'intestin ».

En 1921, d'ailleurs, Rodenwaldt et Roeckemann avaient émis l'idée qu'à la faveur de certaines circonstances pathologiques modifiant l'état de la muqueuse intestinale et la composition de ses sécrétions, il y avait peut-être possibilité d'une réinfestation endogène. 
L'hypothèse de Penso s'appuie sur les arguments suivants : on trouve fréquemment des femelles gravides dans l'épaisseur même de la muqueuse du tube digestif de l'homme (cf. la première partie de cette note). Dans certains cas, des œufs peuvent même être trouvés (3). D'une façon analogue, dans la muqueuse de certaines espèces animales parasitées par des Oxyuridés voisins de celui de l'homme, peuvent s'observer des femelles gravides, des œufs embryonnés à divers stades, des coques vides, des larves très jeunes : femelles gravides dans la muqueuse intestinale du chimpanzé (Weinberg, 1904), larves de Dermatoxys veligera dans la muqueuse

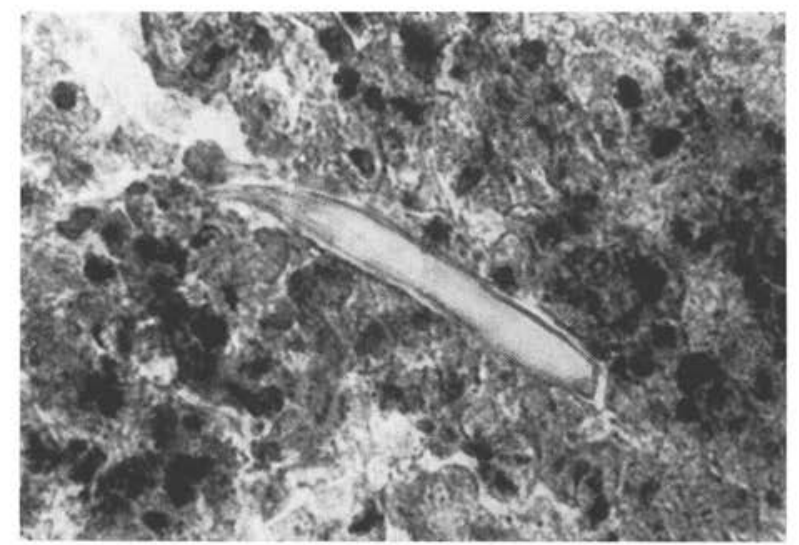

Рнот. 5. - Restes cuticulaires d'une jeune larve

du lièvre (Wetzel, 1931) (4), d'œufs (mais pas de larves) de Passalurus ambiguus dans la muqueuse de cette même espèce (Penso, 1932 et 1933).

Cette théorie fut reprise, par la suite, par Lopez-Neyra (1940). Cet auteur aurait obtenu, expérimentalement, les différentes phases évolutives de l'Oxyure en appendices ligaturés et maintenus in vitro à $35^{\circ} \mathrm{C}$.

Vucetich et Giacobbe (1949) admettent eux aussi la possibilité d'un cycle entièrement intra-intestinal. Dans plusieurs appendices

(3) Concernant ces observations, Penso ne précise pas à quel stade d'embryonnement étaient parvenus les œufs trouvés dans l'épaisseur de la muqueuse d'appendices humains (1932). Il précise par contre (1933) n'avoir jamais rencontré, sur coupes histologiques, d'embryons libres.

(4) Wetzel d'ailleurs avait interprété la présence de larves très jeunes dans l'épaisseur de la muqueuse comme résultant d'une migration en provenance de la lumière de l'intestin et non, comme Penso, d'une éclosion in situ d'œufs déposés là par des femelles gravides. 
humains, en effet, ils observent dans le contenu et le produit de grattage de la muqueuse des œufs à tous les stades de développement : segmentés (2, 4 blastomères, ou plus), embryonnés, avec larves vermiformes prêtes à sortir. Ils trouvent même des larves libres et ceci, parfois, en grande quantité : « ...les cas 31 et 54 sont également extrêmement convaincants, puisque nous y avons rencontré une quantité extraordinaire de larves libres... ».

Il est possible cependant que ce cycle interne n'ait pas toujours lieu et de nombreux auteurs nient même sa possibilité :

- parce que eux-mêmes n'ont pu retrouver d'œufs embryonnés ni de larves venant d'éclore ;

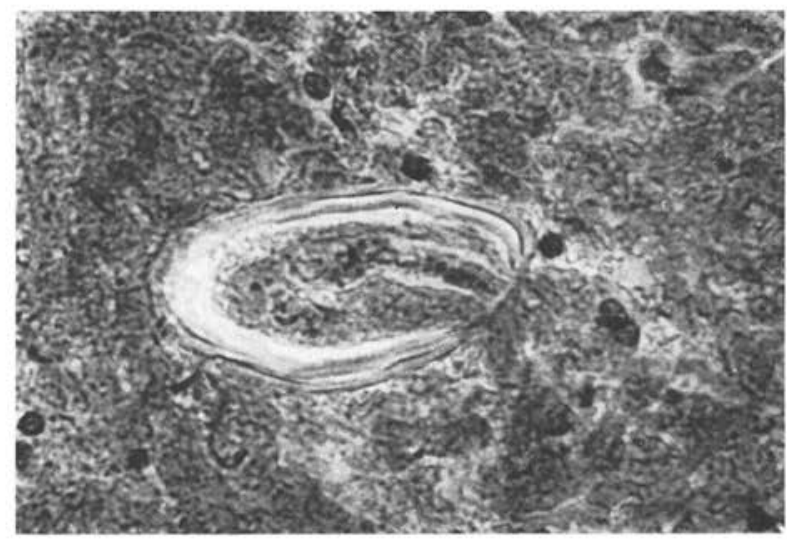

Рнот. 6 - Euf envahi, au niveau de l'aire poreuse, par des cellules du tissu réactionnel environnant, avant mème que l'embryon n'en soit sorti.

- parce que l'œuf, pour atteindre le stade larviforme infestant et pouvoir éclore, devrait être soumis à certaines conditions ne se rencontrant pas dans l'épaisseur de la muqueuse ;

- parce que l'on ne rencontre pas, le plus souvent, de femelles à maturité gravidique suffisante ailleurs que dans les toutes dernières portions du tube digestif.

Ainsi, Madsen (1945) observe des œufs dans la paroi de l'intestin, mais jamais ayant atteint le stade gyriniforme. De même, Smalt (1946, in Symmers, 1950) est incapable de trouver des stades de développement d'Oxyures s'effectuant dans les tissus de l'hôte. Enfin, Deblock (1952), après l'examen de 132 appendices extirpés chirurgicalement, dont plus de la moitié présentait des Oxyures dans 
leur lumière (avec une proportion notable de femelles gravides), signale n'avoir observé qu'une seule fois trois formes larvaires. Et encore, ces larves avaient déjà atteint un stade de développement assez avancé, bien que n'ayant pas encore subi leur mue définitive. Quant aux œufs, cet auteur n'en a jamais vu embryonnés, libres, ni de coques vides, que ce soit dans la lumière appendiculaire ou au sein de la muqueuse elle-même : «Les rares cas où les œufs furent découverts provenaient manifestement de femelles traumatisées pendant l'opération ou sectionnées accidentellement au cours de l'ouverture de l'appendice au laboratoire. »

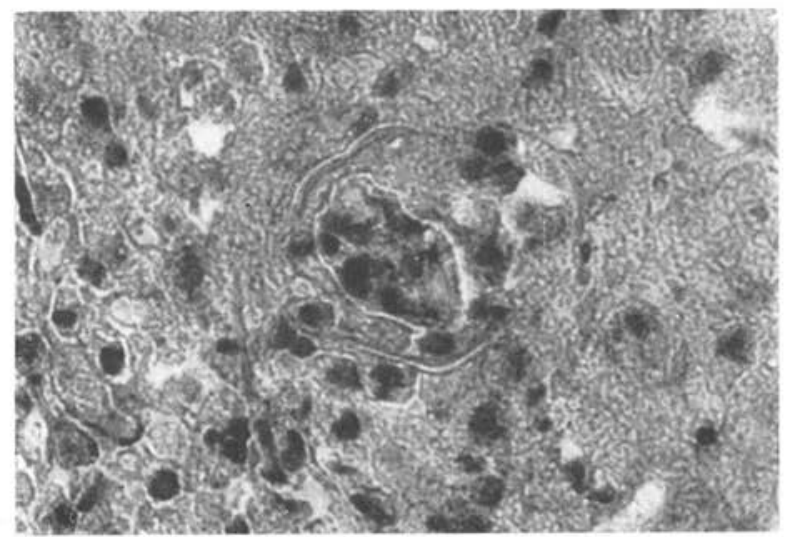

Рнот. 7. - Restes fragmentés de la coque d'un œuf envahi par des cellules environnantes

Pour d'autres, il n'y aurait pas possibilité d'évolution directe pour l'œuf éventuellement pondu dans l'épaisseur de la muqueuse. Celui-ci, pour éclore, devrait subir préalablement l'action des sucs digestifs.

Certes, expérimentalement, l'éclosion est très facilement obtenue en soumettant les œufs aux sucs digestifs : Wundt (1924) l'obtient par action de liquide gastrique naturel ; Cram (in Jones et Nolan, 1942), par contact stomacal ou duodénal; Brumpt (1949), par contact duodénal; Schüffner et Swellengrebel (1949), par action de suc gastrique artificiel. Mais, contrairement à l'opinion de Wundt, qui ne pense pas que l'infestation sans passage par la bouche et l'estomac puisse se faire, l'éclosion de l'œuf n'exige pas la présence de ferments digestifs. En effet, un simple contact avec de l'eau pure peut être suffisant: Philpot (1924) obtient l'éclosion in 
vitro après un séjour de 70 heures, à $22^{\circ} \mathrm{C}$. De même, Zawadowsky et Shalimov (1929) observent que les œufs ayant atteint le stade gyriniforme sont capables de se développer dans l'eau pure, dans le sérum physiologique et dans diverses autres solutions salines. Plus récemment, Deschiens (1944) obtient le développement par un séjour de 5 à 20 jours en chambre humide à la température du laboratoire et l'éclosion par immersion pendant 10 à 15 minutes dans de l'eau distillée à $37^{\circ} \mathrm{C}$.

Par ailleurs, la présence d'oxygène serait également nécessaire au développement de l'œuf. Cette opinion est soutenue par Zawadowsky et Shalimov. D'autres estiment, comme Koch (1928, in Biguet, 1949), que cette présence n'est pas indispensable. Certains, d'ailleurs, dont les deux auteurs russes précités, de même que Madsen (1945), pensent que, éventuellement, les œufs ponçus dans l'épaisseur de la muqueuse peuvent trouver l'oxygène nécessaire à partir du sang.

Enfin, l'infestation endogène serait impossible en raison de la maturation gravidique insuffisante des femelles localisées ailleurs que dans les toutes dernières portions du tube digestif. Ainsi, Geller (1946) note que :

- seules, les femelles d'Enterobius vermicularis et de Passalurus ambiguus migrant au rectum présentent dans leur utérus des œufs à maturité capables d'évolution ultérieure (stade gastrula pour Passalurus, larviforme pour Enterobius);

- les vers entraînés passivement par les matières renferment des œufs incapables d'évoluer dans le milieu extérieur. Ceux éliminés par purgation ne renferment que des œufs non segmentés, ou aux tout premiers stades de segmentation. Cette segmentation ne s'effectue que dans la partie inférieure du tube digestif, et les stades capables de se développer dans le milieu extérieur ne se rencontrent que dans la région périanale.

Pour ces raisons, Geller conclut que l'auto-infestation endogène ne se produit pas dans l'oxyurose.

Des constatations analogues, suivies des mêmes conclusions, avaient antérieurement d'ailleurs été faites en partie par Zawadowsky et Shalimov (1929), qui avaient expérimentalement observé que les œufs ne peuvent poursuivre leur évolution après avoir quitté le corps que s'ils ont atteint le stade gyriniforme, et par Madsen (1945), qui avait noté que les seuls œufs capables d'évolution ultérieure sont ceux des femelles trouvées dans le rectum et surtout de celles sorties spontanément de l'anus.

L'examen détaillé de nos coupes histologiques, révélant, comme 
il a été dit plus haut, la présence d'œufs à maturité, d'embryons libres et même de jeunes larves (5), nous permet de confirmer la possibilité d'un cycle endogène d'Enterobius vermicularis, sans qu'il y ait nécessité d'une sortie de l'œuf hors du tube digestif et d'un séjour de plusieurs heures au contact de l'air extérieur pour l'acquisition du pouvoir infectant, ni d'un contact avec des sucs digestifs (6) pour qu'il y ait éclosion et développement ultérieur des larves.

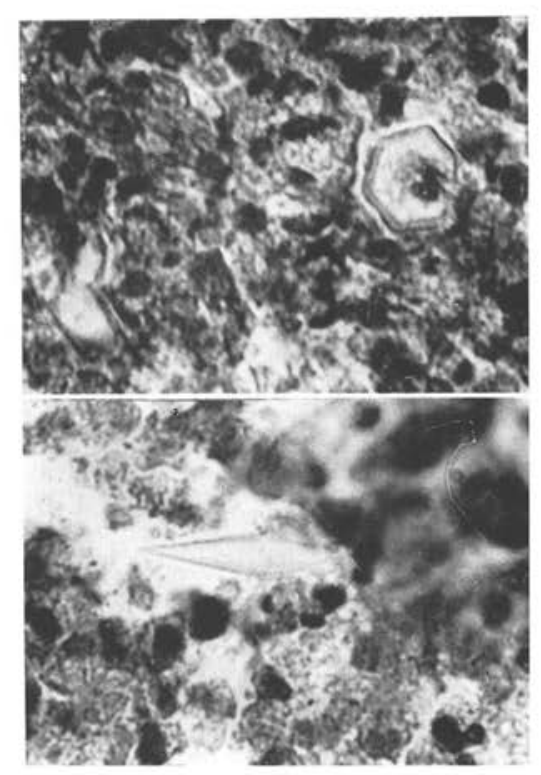

Рнот. 8. - Cristaux de Charcot-Leyden : coupés transversalement en haut, longitudinalement en bas (en losange à extrémités effilées et à section hexagonale).

Toutefois, le fait qu'un cycle évolutif se révèle possible, même dans des conditions naturelles, n'implique pas pour autant son caractère normal, et il est vraisemblable, compte tenu des observa-

(5) Nous n'avons trouvé ces larves que dans les zones de nécrose, jamais dans les tissus sains environnants. Même si de telles larves sont capables de migration, il semble évident que leurs possibilités évolutives hors du tube digestif ne peuvent être que très limitées.

(6) Il semble d'ailleurs que l'action des sucs digestifs, favorisant la sortie de l'embryon, puisse être remplacée ici par l'action lytique du tissu réactionnel environnant. En effet, on observe, à l'extérieur de plusieurs œufs, des amas de cellules groupées au niveau de l’aire poreuse. La pénétration de ces cellules à ce niveau s'effectue parfois alors que l'embryon larviforme est encore à l'intérieur. 
tions précitées de Zawadowsky et Shalimov, de Madsen et de Geller, que le cycle endogène ne se réalise qu'à l'occasion de circonstances très particulières. De toute façon, même si des observations ultérieures venaient à confirmer le caractère relativement fréquent de ce cycle, il semble bien que son importance, dans les phénomènes de surinfestation, soit négligeable en regard de celle du cycle classiquement reconnu, c'est-à-dire du cycle avec passage de l'œuf dans le milieu extérieur.

\section{(Laboratoires de Parasitologie et d'Anatomo-pathologie} de la Faculté de Médecine et de Pharmacie de Rennes)

\section{BibLIOGRAPHIE}

Benedetti, in Leuckant (R.), 1876. - Die menschlichen Parasiten und die von ihnen herrührenden Krankheiten, Leipzig, Winter Ed ${ }^{r}$, vol. 2, p. 345.

Biguet (J.), 1949. - Etat actuel de nos connaissances sur la biologie de l'oxyure, Enterobius vermicularis (Linné, 1758) et sur l'épidémiologè de l'oxyurose. Bull. Soc. Pharmacie de Lille, $\mathrm{n}^{\circ}$ 2, 13.

Bislmer (J), 1946. - An exceptional case of oxyuriasis of the intestinal wall. $J l$ of Parasitology, 32, 359.

Bozicewich (J.) et Brady (F.J.), 1938. - A study of five hundred and four boys in a Brog's camp. Wed. Amer. District of Columbia, 7, 187.

Brady (F.J.) et Wright (W. H.), 1939, - The symptomatology of oxyuriasis as based on physical examinations and case histories of 200 patients. Am. Jl Med. Soc., 198, 367.

Brumpt (E.), 1949. - Précis de Parasitologie, Masson Ed., Paris.

- et LECÈNE, 1909. - Un cas d'appendicite vermineuse ; présence d'oxyures dans la paroi de l'appendice. Bull. Soc. Med. Hop. de Paris, 5 fév.

Cauchemez (L.), 1929. - Un cas remarquable d'appendicite à oxyures. Ann. Parasit. hum. et comp., 7, 280.

Chevrel. (M.-L.), Doey (J.-M.), Dauleux (L.) et Louvet, 1961. - Etude histopathologique des lésions de l'oxyurose génitale chez la femme. Ann. Anatom. Pathol., sous presse.

Chlari (H.), 1928. - Ueber das Vorkommen von Oxyuren in menschlichen Eileiter. Virchows Arch. f. Path. Anat. u. Physiol., 269, 730.

Coutelen (F.), 1950. - Rapport sur le parasitisme intestinal chez les enfants d’âge scolaire. Congrès international de l'amibiase et des parasitoses intestinales de l'adulte el de l'enfant, Châtel-Guyon, sept.

Deblock (S.), 1952. - Contribution à l'étude de loxyurose appendiculaire, Thèse de doctorat en Pharmacie, Lille.

Deschiens (R.), 1944, - Sur les conditions expérimentales d'évolution et d'éclosion des œufs d'oxyuridés. Bull. Soc. Path. exot., 37, 310.

Froriep (E.), 1938. - Zentralbl. $f$. Gynäk., 62, 923, in Symmers, 1950.

Geller (E. R.), 1946, - Analysis of the population of Enterobius vermicularis in various portions of the host's intestine, and autoinvasion in entero- 
biasis (en russe). Med. Parasit. a. Parasitic Dis., Moscow, 15, 45, in Trop. Dis. Bull., 1947, 4h, 927.

Heller (A.), 1903, - Ueber Oxyuris vermicularis, Dtsch. Arch. f. Klin. Med., $77,21$.

JACOBS (A. H.), 1942. - Enterobiasis in children : incidence, symptomatology and diagnosis with a simplified scotch cellulose tape technique. $J l$ Pediatrics, 21, 497.

Jones (M. F.) et Noi.an (M. O.), 1949. - In Biguet, 1949.

Koch (E. W.), 1925. - Oxyuren fortpflanzung im Darm ohne Reinfektion und Magenpassage. Zentralbl. f. Bakt., I, Arb. orig., 94, 208.

Kolb (R.), 1902. - Ueber den Befund von auf dem Peritoneum des Cavum Douglasii angewachsenen Oxyuriden. Zentralbl. f. Bakt. und Parasit., $31,268$.

Liengme (A.), 1932. - De l'appendice vermiforme à coprostase et de l'appendicite à oxyure. Arch. internat. de Méd. Expérim., 7, $\mathrm{n}^{\circ} 4$.

Lopez-Nerra (C. Rodriguez), 1940. - Helmintiasis humana, Manuel de Médecine pratique, Barcelone, Salvat $\mathrm{Ed}^{\mathrm{r} *}$.

Lonentz (F.H.), 1925. - Eine einfache Oxyuren-behandlung. Med. Klin., 21, 95.

MAdSEN (H.), 1945. - Biological observations upon Enterobius vermicularis. Acta path. et Microb. Scandinavica, 22, 392.

Marro (G.), 1901. - Sopra una cisti impiantata sulla salpinghe contenente uova di Oxyuris vermicularis. Arch. per le Scienze mediche, 25, 161.

Penso (G.), 1932. - Présence des œufs d'oxyures en pleine muqueuse intestinale et biologie des oxyures. Ann. Parasit. Hum. et comp., 10, 271.

1933. - Nouvelles considérations sur la biologie des oxyures. Ann. Parasit. hum. et comp., 11, 268.

Pнilpot (F.), 1924. - Notes on the eggs and early development of some species of oxyurida. Jl of Helminthology, 2, 239.

Quintanar (E.), 1943. - Investigacion sobre Enterobius vermicularis en la region vulvar. Rev. del Inst. de Salubr. y Enferm. tropic., 4, 179.

Railliet (G.), 1911. - Les vers intestinaux dans la pathologie infantile, Thèse de Médecine, Paris.

Rodenwaldt (E.) et Roeckemann (W.), 1921. - Zur Biologie von Oxyuris vermicularis. Zentralbl. f. Bakt., I. Arb. Orig., 86, 421.

Schneiner (P.), 1904. - Oxyuris vermicularis im Beckenperitoneum eigekapselt. Zentralbl. f. Bakt., 36, 550.

SchürfNer (W.), 1947. - Experimentelle Infektionen mit Staubeiern von Oxyuris (Enterobius) vermicularis. Zentralbl. f. Bakt., I. Arb. Orig., 152,67 .

1949. - Retrograde Oxyuren-Infektion. « Retrofektion ». IV. Mitteilung. Zentralbl. f. Bakt., I. Arb. Orig., 154, 220.

et Swellencirebel. (N. H.), 1949. - Retrofection in Oxyuriasis. A newly discovered mode of infection with Enterobius vermicularis. Il of Parasitology, 35, 138.

Srmons (E. M.), 1899, - Entozoen in der Gebärmutter. Zentralbl. f. Gynäk., $23,777$.

Smalt (F.H.), 1946, - Nederl. Tijdschr. v. Geneesk, 90, 333, in Symmers, 1950.

SpItzer (B. B. R.), 1892. - Wien. med. Wehnschr., 42, 6, in Symmers, 1950.

Strada (F.), 1907. - Sulla presenza di oxyuride incapsulati nella cavita peritoneale. Arch. per le Scienze med., 31, 418.

Symmers (W. St. C.), 1950. - Pathology of oxyuriasis, with special reference 
to granulomas due to the presence of Oxyuris vermicularis (Enterobius vermicularis) and its ova in tissues. A.M.A. Arch. Path., 50, 475.

- 1957. - Two cases of eosinophilic prostatitis due to metazoan infestation (with Oxyuris vermicularis and with a larva of Linguatula serrata). J. Path. and. Bact., 73, 549.

Troisier (J.) et Deschiens (R.), 1930. - Deux cas d'oxyurose chez le chimpanzé ; traversée de la paroi intestinale jusqu'au péritoine. Ann. Parasit. hum. et comp., 8, 562.

Tschamer (F.), 1919. - Helminths in female genital organs. Zentralbl. $f$. Gynäk., 43, 989.

VAughan (R. A.), 1880-81, - St-Louis Clin. Rec., 7, 271, in Symmers, 1950.

VIX (E.), 1860. - Ueber Entozoën bei Geisteiskranken, insbesondere über die Bedeutung, das Vorkommen und die Behandlung von Oxyuris vermicularis. Allgemeine Zeitschrift für Psych., 17, 1149.

Vucetich (M.) et GincobBe (O.), 1949. - Parasitologia de appendices extirpados en la ciudad de Jujuy (R.A.). Ann. Inst. Med. Reg., 2, 245.

Vuillemin (P.), 1902. - Sur la pénétration des femelles d'Oxyuris vermicularis à travers les parois de l'intestin. Zentralbl. f. Bakt. u. Parasiten., Abt 1, 32, 538.

Weinberg (M.), 1904, - Un cas d'appendicite chez le chimpanzé. Ann. Inst. Pasteur, 18, 323.

WETzEL (R.), 1931. - On the biology of the fourth-stage larva of Dermatoxys veligera (Rudolphi 1819), Schneider 1866, an oxyurid parasitic in the hare. $\mathrm{Jl}$ of Parasit., 18, 40.

Whitelocke (A.), 1914. - Cas simulant la méningite, dans lequel les symptômes furent causés par l'irruption dans le péritoine d'oxyures à travers l'appendice. Ann. Arch. Mal. Enfants, 382.

Wundr (N.), 1924. - Ueber Möglichkeit der intraintestinalen Entwicklung von Oxyuren unter Umgehung der Magenpassage. Muench. Med. Woch., $71,546$.

Zawadowsky (M. M.) et Shalimov (L. G.), 1929. - Die Eier von Oxyuris vermicularis und ihre Entwicklungs-bedingungen, sowie ueber die Bedingungen, unter denen eine Autoinfektion bei Oxyuriasis unmöglich ist. Ztschr. f. Parasitenk., 2, 12.

(Microphotographies du Laboratoire de Parasitologie) 